\title{
Nouvelle approche pour la caractérisation du dévissage et desserrage des assemblages vissés
}

\author{
Christophe Delcher* \\ CETIM, Pôle IDA - Ingénierie Des Assemblages, 7 rue de la Presse, 42952 Saint-Étienne Cedex 9, France
}

Reçu le 3 novembre 2017 / Accepté le 21 août 2018

\begin{abstract}
Résumé. Différents tests permettant d'évaluer la performance au desserrage des assemblages vissés existent. Néanmoins, leurs protocoles et conditions d'essai sont spécifiques et souvent assez peu détaillés. De ce fait, réaliser des tests comparatifs répétables, en particulier inter-laboratoires, devient rapidement délicat et sujet à controverse. Dans le cadre de la commission R\&D Fixations regroupant les fabricants français de fixations et suite aux études menées sur le sujet du dévissage spontané menées par le CETIM et ARTEMA Fixations (anciennement AFFIX), des travaux sont actuellement en cours afin de normaliser (norme expérimentale française) une méthodologie générale pour la réalisation d'essai de desserrage quel qu'il soit. Le présent document intègre une première partie exposant un panorama des différents tests de desserrage existants. Une seconde partie détaille la nouvelle approche de caractérisation du seuil de desserrage, applicable à tous types de tests déjà existants.
\end{abstract}

Mots clés : fixations / assemblages vissés / desserrage / serrage / précharge

\begin{abstract}
New approach for the loosening charachterization of bolted assembly. Various tests are available to evaluate the loosening performance of bolted assemblies. However, the protocols and conditions for these tests are specific and often not very detailed. As a result, conducting repeatable comparative tests (especially inter-laboratory and round robin) quickly becomes delicate and controversial. The "R\&D Fixations" commission of the CETIM (technical center of the French mechanical industries) gathering the French manufacturers of fasteners and its union (ARTEMA Fixations) carried out many works on the spontaneous unscrewing and loosening since for more than ten years. The work currently in progress consists in standardizing (French experimental standard) a general methodology for carrying out any kind of loosening test. This document includes a first part presenting an overview of the various existing loosening tests. A second part details the new approach for characterizing the loosening threshold, applicable to all types of tests that already exist.
\end{abstract}

Keywords: fastener / bolted assembly / loosening / tightening / preload

\section{Introduction}

Les liaisons vissées et boulonnées sont des solutions d'assemblage très largement répandues et utilisées dans tous les domaines industriels. Ce succès est principalement lié à leur facilité de mise en œuvre et à leur aptitude à assembler des matériaux différents. Elles présentent en outre une tenue mécanique élevée et une démontabilité plus aisée comparées aux autres technologies d'assemblage, pour un coût relativement faible.

Néanmoins, lorsqu'elles sont utilisées en tant qu'assemblage structurel fortement sollicité, il convient de veiller à leur qualité de conception, leur dimensionnement et leur

\footnotetext{
* e-mail: christophe.delcher@cetim.fr
}

facilité et fiabilité de mise en œuvre. En effet, outre les problématiques de fabrication des éléments de fixation qui requièrent un savoir-faire certain, deux phénomènes sont fréquemment les causes de défaillance:

- la non-tenue de la liaison au dévissage et au desserrage; - la non-tenue de la liaison soumise à des sollicitations dynamiques (fatigue).

La bonne tenue d'un assemblage vissé ou boulonné soumis à des sollicitations dynamiques est, d'une manière générale, directement liée à un niveau de serrage suffisamment élevé. Ce dernier étant en particulier assuré par une mise en œuvre maîtrisée. Sa tenue peut être calculée grâce aux modèles analytiques existants [12-17], ou numériquement grâce aux méthodes par éléments finis $[14,17]$. 
Tableau 1. Essais normalisés de résistance au dévissage/ desserrage.

Table 1. Standardised unscrewing/loosening resistance tests.

\begin{tabular}{ll}
\hline Référence - Norme & Type de sollicitations \\
\hline $\begin{array}{l}\text { ISO 16130 } \\
\text { (Junker DIN 65 151, }\end{array}$ & $\begin{array}{l}\text { Sollicitation transversale } \\
\text { par mouvement alterné }\end{array}$ \\
$\begin{array}{ll}\text { NIN 25201-4) } \\
\text { ISO 7450 / NASM 1312-7 }\end{array}$ & $\begin{array}{l}\text { Sollicitation transversale } \\
\text { par vibrations et chocs } \\
\text { (verticaux) }\end{array}$ \\
NF E 25-005 & $\begin{array}{l}\text { Sollicitation transversale } \\
\text { par chocs (horizontaux) }\end{array}$ \\
\hline
\end{tabular}

Concernant les phénomènes de dévissage et de desserrage, ils restent encore assez mal connus et difficilement maîtrisés.

Ces deux phénomènes sont définis de la manière suivante :

- desserrage (ou relâchement): perte de tension sans mouvement relatif vis/écrou, occasionnée par les phénomènes de tassement, matage ou fluage et relaxation;

- dévissage: perte de tension causée par un mouvement relatif angulaire vis/écrou (rotation d'un élément par rapport à l'autre).

Le présent document dresse un panorama des différents tests de dévissage/desserrage existants et synthétise les travaux de normalisation en cours concernant une nouvelle approche pour la caractérisation du seuil de desserrage, applicable à tous types de tests déjà existants.

\section{Panorama des différents tests de dévissage/desserrage existants}

Il existe différents types d'essais pour caractériser la résistance au dévissage/desserrage des assemblages vissés. Qu'ils soient normalisés ou non la grande majorité génère principalement, au niveau de la fixation, des sollicitations transversales (cisaillement) par mouvements, vibrations ou chocs.

\subsection{Les essais normalisés}

Le tableau suivant (Tab. 1) récapitule les différents essais normalisés existants de résistance au dévissage/desserrage ainsi que le type de sollicitations qu'ils génèrent.

Les essais les plus communément pratiqués sont les essais suivant la norme ISO 16130 (essai «Junker» [3]) ainsi que l'essai de vibration suivant les normes NAS 3350 / NASM 1312-7.

Dans tous les domaines, les essais «Junker» sont souvent réalisés pour leur simplicité et leur coût relativement faible. Les essais NAS 3350 / NASM 1312-7 sont des essais particulièrement rencontrés dans le domaine aéronautique pour la qualification des systèmes d'anti-dévissage des fixations à pas impérial. Les essais suivant les normes ISO 7481 et ISO 8642 sont identiques aux essais NAS / NASM mais pour les fixations à pas métrique.
De même, les essais NF E 25-005 sont similaires aux essais NAS/NASM, mais la sollicitation est appliquée horizontalement par une machine rotative à excentrique et non pas verticalement par un pot vibrant.

L'essai ISO 16130 «Junker» consiste à solliciter la fixation à tester par un mouvement transversal alterné. Le dispositif d'essai est composé d'un plateau mobile assemblé à un support fixe par la fixation à tester. Un moteur rotatif applique le mouvement transversal alterné au support mobile via un excentrique, voir figure 1 . Les paramètres suivants peuvent être réglés ou ajustés:

- la fréquence (entre 10 et $15 \mathrm{~Hz}$ suivant la norme ISO 16130);

- le déplacement du plateau mobile.

Les essais NAS 3350 / NASM 1312-7 et ISO 7481 et 8642 consistent à solliciter la fixation à tester par des vibrations et chocs transversaux. Le dispositif d'essai est composé d'un pot vibrant supportant un montage d'essai disposant de trous oblongs. Les fixations à tester sont assemblées sur des entretoises laissées libres dans les trous oblongs, voir figure 2. Le pot vibrant applique des vibrations qui génèrent des chocs entre le montage d'essai et les entretoises. La fréquence (environ $30 \mathrm{~Hz}$ ) et le déplacement (environ 11,4 $\mathrm{mm}$ ) sont définis.

L'essai NF E 25-005 est similaire à l'essai NAS / NASM, il consiste à solliciter la fixation à tester par des chocs. Le dispositif d'essai est composé d'un plateau mobile assemblé à un support fixe par la fixation à tester. Un moteur rotatif applique le mouvement transversal alterné au support mobile via un excentrique, voir figure 3. La fréquence (environ $22 \mathrm{~Hz}$ ) et le déplacement (environ 22,8 mm) sont définis.

\subsection{Les essais non normalisés}

Différentes études expérimentales sur le dévissage spontané ont été réalisées pour des configurations de sollicitations purement axiales. On peut citer en particulier les travaux initiés par Goodier et Sweeney (1945) [4], les travaux de Gambrell (1968) [5] et de Hess et Davis (1996) [6].

Néanmoins, ces travaux montrent que ce type de sollicitations génère difficilement du dévissage spontané.

Quelques études ont pu être réalisées pour des configurations de sollicitations en torsion, représentant essentiellement des assemblages fonctionnant par adhérence et disposant d'une seule fixation (exemple: fixation de poulie, de barre de torsion...). On peut citer en particulier les travaux de Clark et Cook (1966) [7], voir figure 4, et Sakai (1978) [8].

La grande majorité des études expérimentales sur le dévissage spontané ont été réalisées pour des configurations de sollicitations transversales avec chocs, vibrations ou non.

On peut citer en particulier les travaux de Gerhard H. Junker (1969) [3] qui ont par la suite été intégrés à la norme DIN 65151 (voir Sect. 2.1).

De même, les travaux suivants peuvent être évoqués:

- les travaux de Pearce (1973) [9], qui ont utilisé une machine d'essai comparable mais générant des sollicitations par chocs (Fig. 5); 


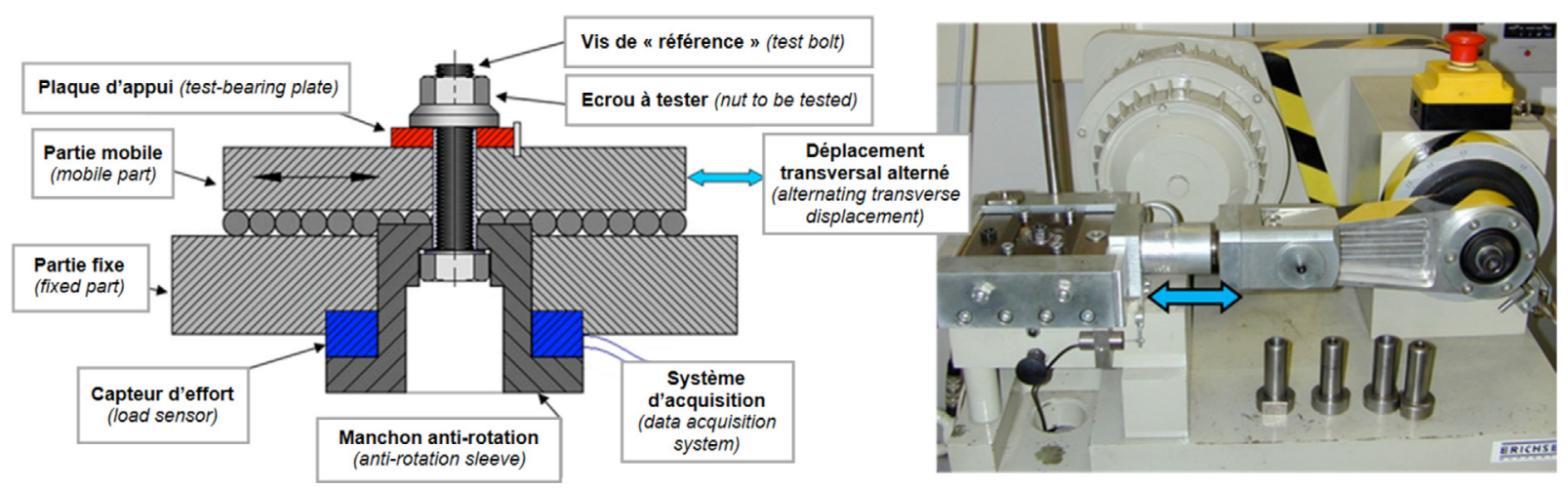

Fig. 1. Schéma dispositif d'essai ISO 16130 « Junker».

Fig. 1. ISO 16130 "Junker" test device diagram.
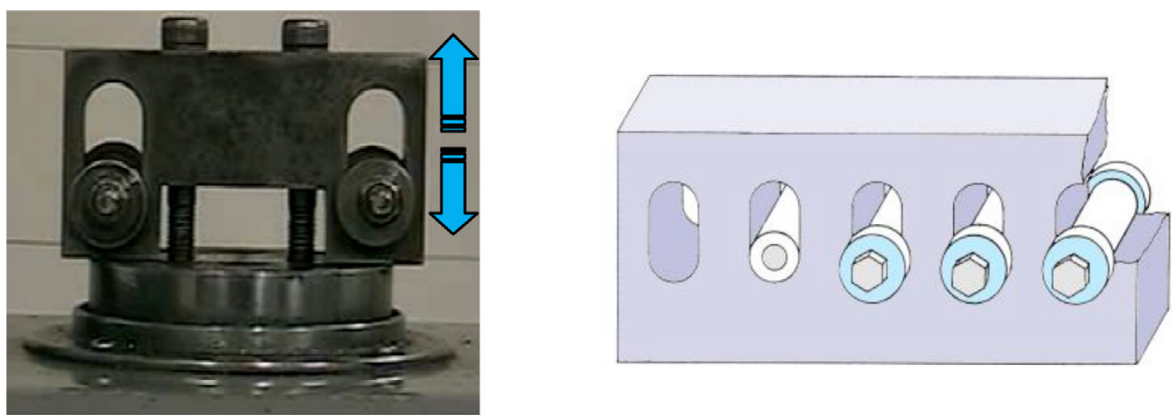

Fig. 2. Schéma dispositif d'essai NAS/NASM.

Fig. 2. NAS/NASM test device diagram.

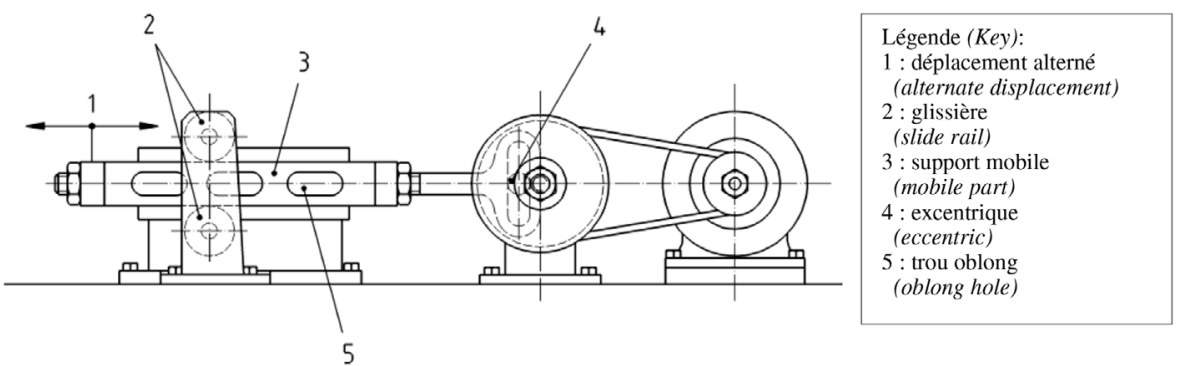

Fig. 3. Schéma dispositif d'essai NF E 25-005.

Fig. 3. NF E 25-005 test device diagram.

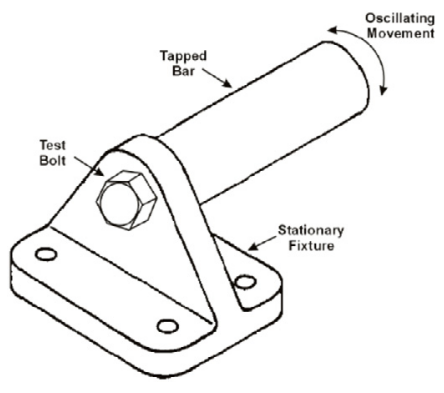

Fig. 4. Schéma dispositif d'essai de Clark et Cook.

Fig. 4. Clark and Cook test device diagram.

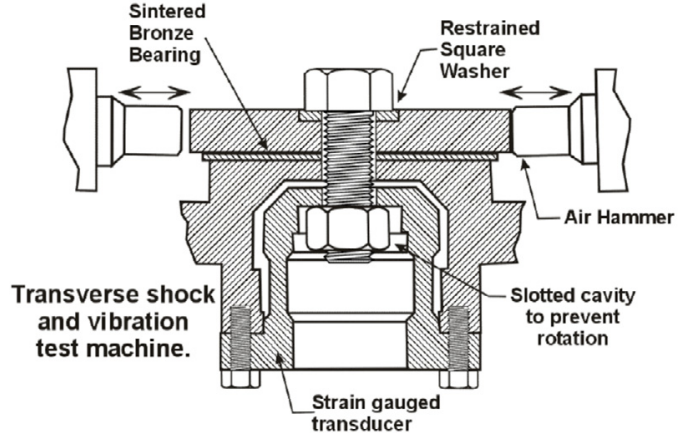

Fig. 5. Schéma dispositif d'essai de Pearce.

Fig. 5. Pearce test device diagram. 


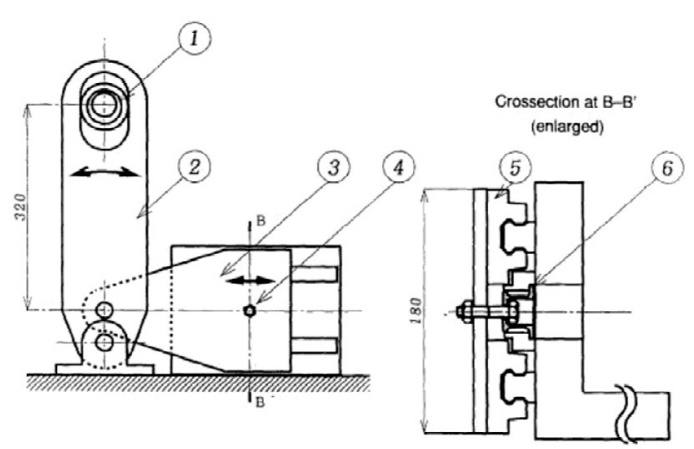

Dispositif par déplacement

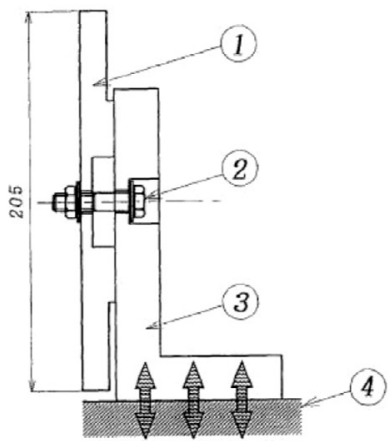

Dispositif par accélération

Fig. 6. Schéma dispositifs d'essai de Sase et al.

Fig. 6. Sase et al. test device diagram.

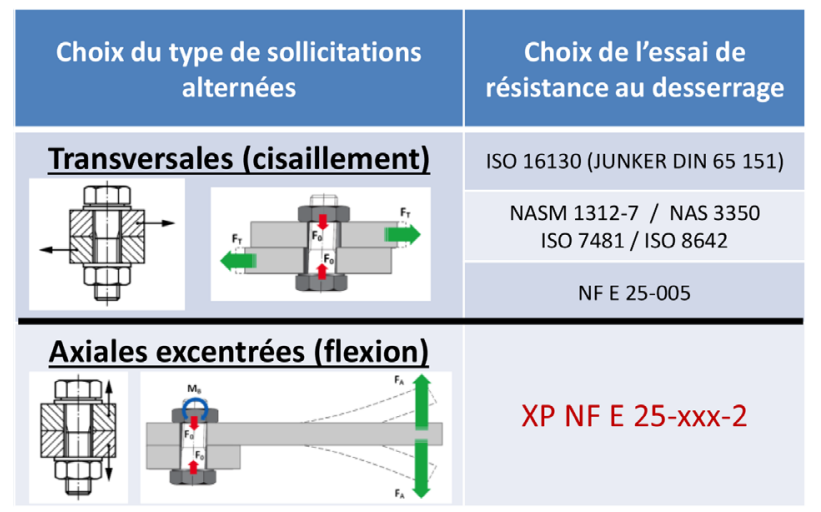

Fig. 7. Ensemble des essais normalisés et type de sollicitations.

Fig. 7. Standardized set of tests and type of loads.

- les travaux de Sase et al. (1996) [10], qui ont conçu une machine d'essai de desserrage par déplacement et une autre par accélération (Fig. 6).

En parallèle de ces études expérimentales et afin d'améliorer la compréhension du phénomène de dévissage spontané, beaucoup d'études ont été menées sur sa modélisation et son calcul pour des configurations de sollicitations transversales.

\subsection{Essai par flexion alternée en cours de normalisation}

Comme évoqué en début de la section 2, la grande majorité des essais génèrent principalement, au niveau de la fixation, des sollicitations transversales (cisaillement). Ceci s'explique par le fait que ce type de sollicitation est le plus sévère vis-à-vis du dévissage spontané et donc permet de reproduire expérimentalement ce phénomène relativement facilement.

Néanmoins, l'ensemble de ces essais ne permettent pas de reproduire d'autres conditions de sollicitations qui pourraient générer des problématiques de dévissage. Afin de pouvoir caractériser la résistance au dévissage/desserrage dans des conditions de sollicitations différentes, une méthode d'essai de résistance au dévissage par flexion alternée dite «hélicoptère»a pu être mise au point ces dernières années par le CETIM, ARTEMA Fixations et les industriels de la commission R\&D Fixations [1]. Cette méthode est à ce jour en cours de normalisation au niveau français.

La contribution de cette nouvelle méthode d'essai est de proposer un mode de sollicitations plus proche de certaines applications industrielles. La figure 7 permet d'illustrer son positionnement (XP NF E 25-xxx-2) par rapport aux méthodes d'essai déjà existantes, l'intérêt étant de pouvoir choisir le type de sollicitations le plus proche de l'application industrielle étudiée.

Cet essai applique une contrainte de flexion plane alternée au niveau de la fixation testée par la mise en résonance d'une poutre martyre.

L'appareillage d'essai se compose principalement d'un système permettant d'appliquer un déplacement alterné. Ce déplacement peut être généré simplement grâce à un moteur rotatif avec un système bielle manivelle, ou bien par un vérin hydraulique, ou éventuellement avec un pot vibrant (Fig. 8).

Un capteur d'effort est intégré au montage afin de mesurer l'effort de serrage tout au long de l'essai.

Les principales caractéristiques de l'appareillage et du dispositif d'essai sont les suivantes:

- déplacement alterné du support de poutre réglable: \pm 2 $\mathrm{mm}$ au minimum;

- fréquence du déplacement alterné réglable, entre $(10 \mathrm{~Hz}-$ $25 \mathrm{~Hz})$;

- mesure de l'effort de serrage avec une précision de $\pm 2 \%$ sur l'ensemble de la chaîne de mesure étalonnée, comprenant: capteur, câble, amplificateur et système d'acquisition ;

- mesure du déplacement avec une précision de $\pm 3 \%$ sur l'ensemble de la chaîne de mesure étalonnée, comprenant : capteur, câble, amplificateur et système d'acquisition.

L'assemblage subissant de fortes sollicitations lors de l'essai, il est nécessaire d'éviter l'endommagement des interfaces entre : le support de poutre et la poutre martyre, ainsi qu'entre la poutre martyre et la fixation testée. De ce fait, le dispositif d'essai utilise des cales fusibles (Fig. 9) qui permettent de réaliser l'essai dans des conditions reproductibles en particulier vis-à-vis de l'usure des interfaces (Fig. 10). 


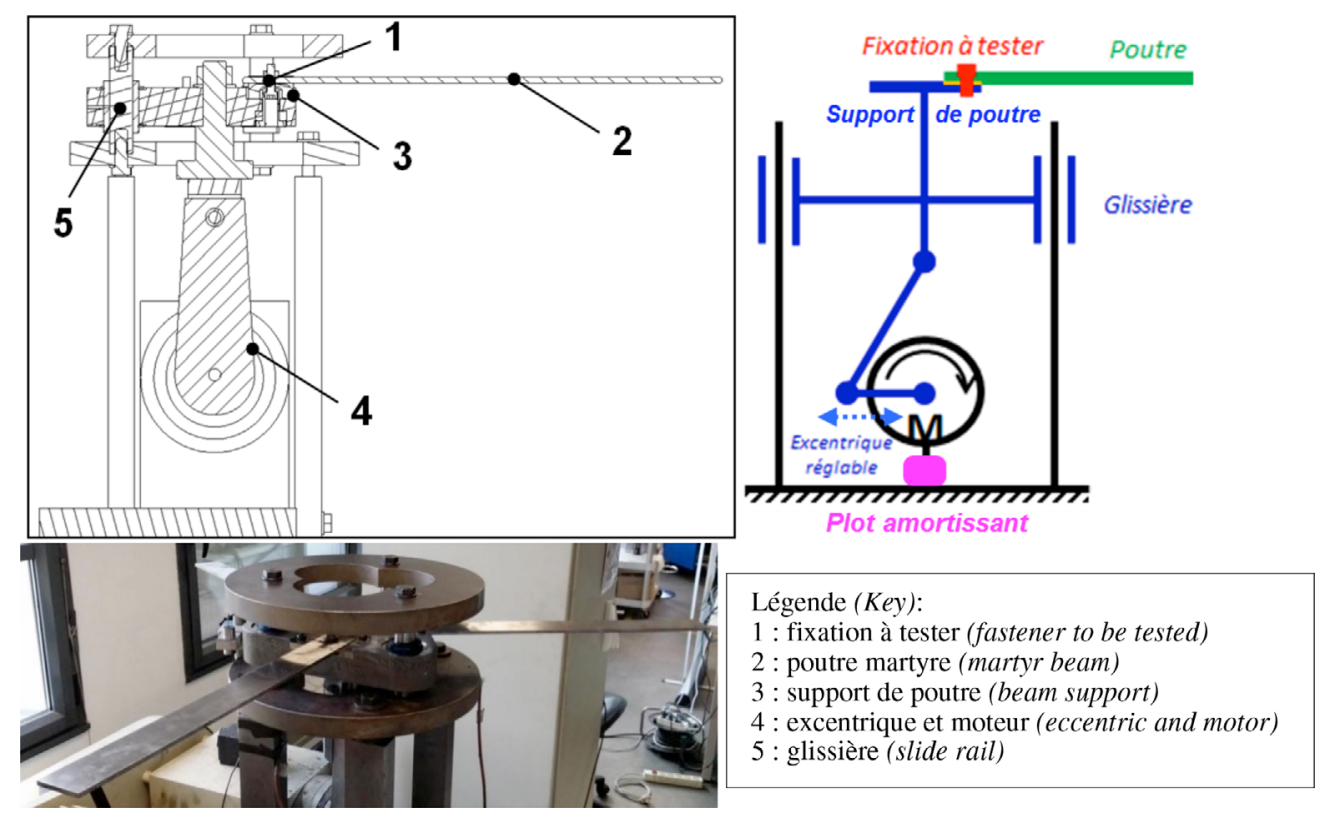

Fig. 8. Schéma banc d'essai de résistance au dévissage/desserrage par sollicitation axiale excentrée.

Fig. 8. Schematic of the test bench for resistance to unscrewing/loosening by eccentric axial loading.

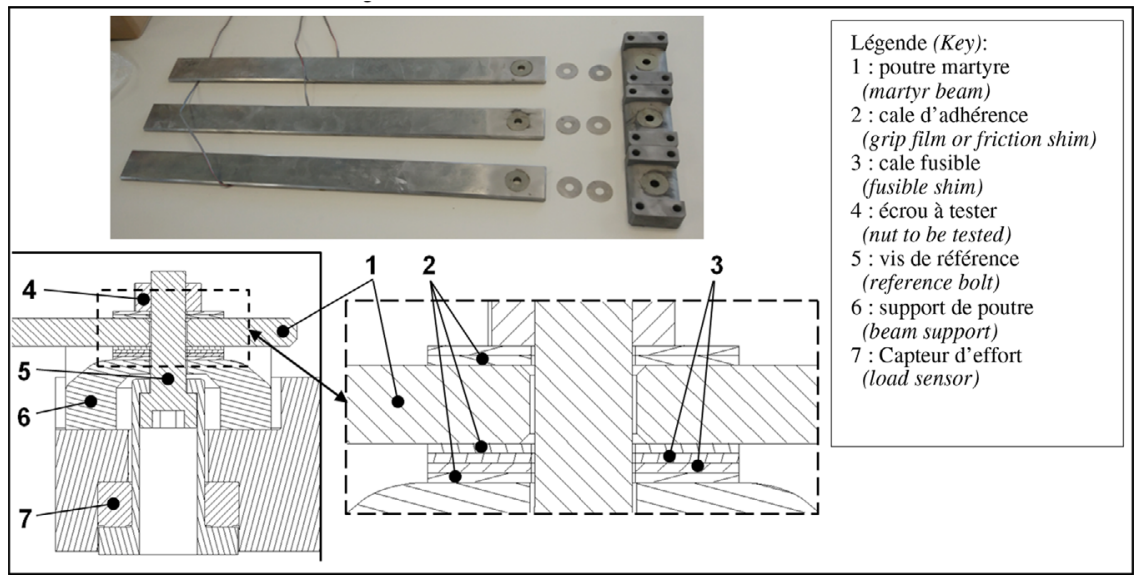

Fig. 9. Schéma dispositif d'essai de résistance au dévissage/desserrage par sollicitation axiale excentrée. Note: la représentation des cales a été intentionnellement surdimensionnée afin de faciliter la lecture de la figure 9 (l'épaisseur réelle de ces cales est de l'ordre de $0,2 \mathrm{~mm})$.

Fig. 9. Schematic of the test device for resistance to unscrewing/loosening by eccentric axial loading.

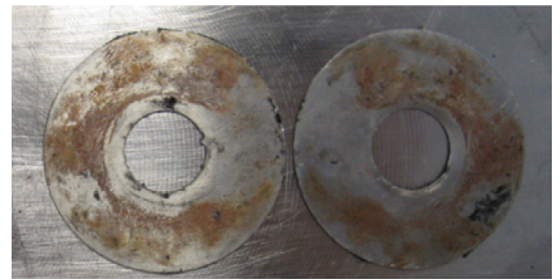

Fig. 10. Cales fusible après essai.

Fig. 10. Fuse shims after test.

\section{Nouvelle approche pour la caractérisation de la résistance au dévissage/desserrage}

L'ensemble des travaux sur le dévissage spontané réalisés ces dernières années par le CETIM, ARTEMA Fixations et les industriels de la commission R\&D Fixations [1], ont de même permis de définir une méthodologie robuste pour caractériser la résistance au dévissage/desserrage.

L'ensemble des essais de dévissage existants apportent des résultats qui sont souvent sujet à controverse car leur méthodologie ne permet pas la plupart du temps d'obtenir 


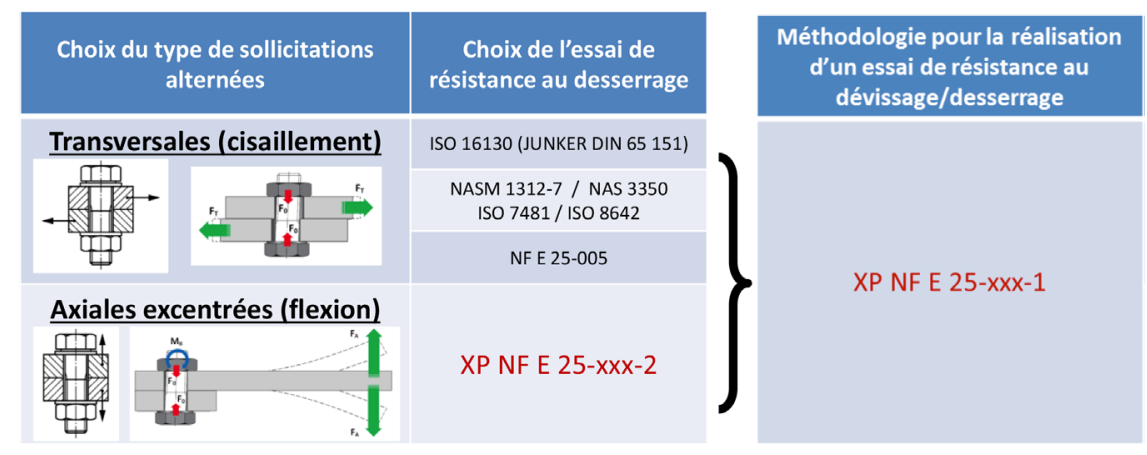

Fig. 11. Ensemble des essais normalisés et méthodologie de détermination de la résistance au dévissage.

Fig. 11. Standard set of tests and methodology for determining unscrewing resistance.

Tableau 2. Méthode «escalier court» pour les essais de dévissage/desserrage.

Table 2. Short staircase method for unscrewing/loosening tests.

\begin{tabular}{|c|c|c|}
\hline \multirow[t]{6}{*}{ Sollicitations } & Niveau de résistance $S_{\mathrm{d}}(\mathrm{N})$ & $\begin{array}{l}\text { Résistance moyenne au dévissage/desserrage } \\
\text { (en mm si le banc ne permet pas un pilotage en effort) }\end{array}$ \\
\hline & Niveau de départ $S_{0}(\mathrm{~N})$ & À déterminer par dichotomie ( $\approx 3$ à 4 échantillons, 5 maximum $)$ \\
\hline & Ecart entre les niveaux $\Delta S_{\mathrm{d}}$ & Demi-intervalle entre les deux derniers essais préliminaires \\
\hline & Nombre de cycles à effectuer, $n$ & $\begin{array}{l}\text { À définir suivant le type d'essai de desserrage choisi } \\
\text { et de l'assemblage testé }\end{array}$ \\
\hline & Fréqu & \\
\hline & Nombre de répétitions & $5(\mathrm{apr}$ \\
\hline \multicolumn{2}{|c|}{ Effort de serrage initial $F_{0}(\mathrm{~N})$} & Effort correspondant au serrage minimal de l'assemblage : $F_{0}=F_{0}$ \\
\hline \multicolumn{2}{|c|}{ Longueur serrée $L_{\mathrm{p}}(\mathrm{mm})$} & $\begin{array}{l}\text { À définir suivant le type d'essai de desserrage choisi } \\
\text { et de l'assemblage testé (par défaut: } L_{\mathrm{p}} \leq 3 d \text { ) }\end{array}$ \\
\hline \multicolumn{2}{|c|}{ Critère de défaillance } & $50 \%$ de perte de la tension initiale de serrage $: F_{0}{ }^{\min } \leq 50 \% F_{0}$ \\
\hline
\end{tabular}

des résultats discriminants et reproductibles entre laboratoires (en particulier lorsque les bancs d'essai utilisés ne sont pas strictement identiques).

En effet, ces méthododologies de caractérisation imposent des niveaux de sollicitations fixes. De ce fait, des fixations ou technologies différentes ayant une bonne résistance au dévissage/desserrage ne pourront pas être comparées efficacement. En effet, elles obtiendront des résultats proches et ne seront jamais mises en défaut par les différents tests existants. De plus, l'ensemble des essais étant dynamiques, la rigidité et l'amortissement des dispositifs d'essai peuvent interagir sur les résultats.

La nouvelle méthododologie de caractérisation permet de déterminer la limite de résistance au dévissage/ desserrage de la fixation testée par une adaptation des méthodes utilisées pour la caractérisation de la limite d'endurance des matériaux en fatigue [11]. Cette approche a déjà pu être investiguée par Shinji KASEI et Minoru SAWAI (1983) [2].

Les travaux réalisés ont permis d'adapter la méthode « escalier court » ou «modified Staircase » [11] aux essais de résistance au devissage/desserrage quels qu'ils soient. Cette méthodologie est, à ce jour, en cours de normalisation au niveau français. La figure 11 permet d'illustrer son positionnement (XP NF E 25-xxx-1) vis-à-vis de l'ensemble des méthodes d'essai.
La résistance au dévissage/desserrage $S_{\mathrm{d}}$ d'une fixation caractérise sa résistance moyenne au dévissage/desserrage pour une tenue à $n$ cycles. Ce paramètre correspond au niveau de sollicitation (exemple: effort, déplacement...) à imposer à la configuration testée pour provoquer la défaillance de $50 \%$ des échantillons avant le $\mathrm{n}^{\mathrm{e}}$ cycle de sollicitations.

La méthodologie est à réaliser en suivant les deux étapes décrites aux sections 3.2 et 3.3 .

\subsection{Paramètres à fixer}

Le tableau 2 présente les étapes et paramètres de la méthode 《escalier court» appliqués aux essais de résistance au dévissage/desserrage. L'ensemble de ces éléments sont détaillés dans les paragraphes suivants.

\subsubsection{Nombre de cycles $n$ à chaque palier}

Le nombre de cycles de sollicitations à effectuer à chaque palier doit être constant pour tous les échantillons testés, il dépend du type d'essai de dévissage/desserrage choisi (Tab. 1).

Pour l'application de la méthode «escalier court » aux essais de dévissage/desserrage, un nombre de cycles relativement faible (quelques centaines à quelques milliers) 

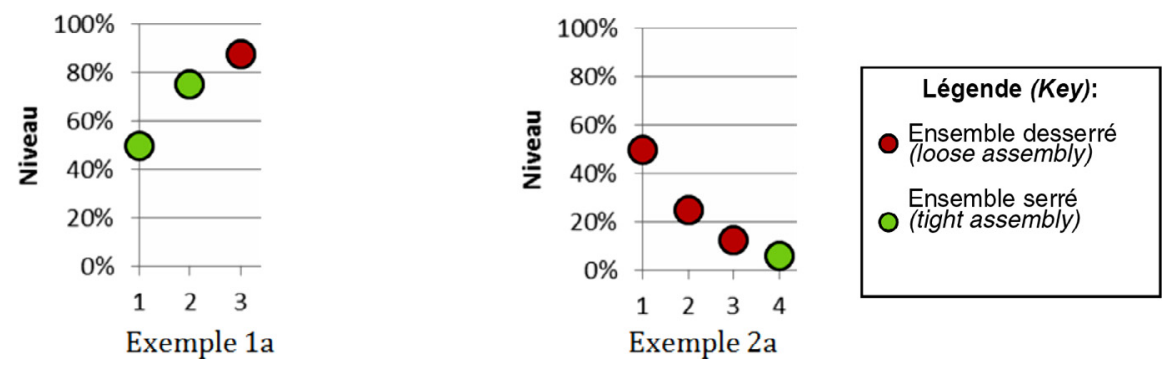

Fig. 12. Étape 1-Recherche du niveau de départ par dichotomie.

Fig. 12. Step 1-Search for the starting level by dichotomy.

est à privilégier car avec cette méthode les essais sont accélérés et sévérisants.

Un nombre de cycles important (au-delà de 10000 cycles) tend à rendre les résultats dispersifs, en particulier liés à des phénomènes d'usure des interfaces des pièces testées (exemple: dégradation des revêtements, fretting...).

\subsubsection{Fréquence des sollicitations}

La fréquence des sollicitations doit être constante pour tous les échantillons testés, elle dépend du type d'essai de dévissage/desserrage choisi et de l'assemblage testé (Tab. 1).

Une fréquence relativement faible est généralement suffisante et privilégiée (de l'ordre de quelques dizaines de hertz).

\subsubsection{Effort de serrage initial}

L'effort de serrage initial doit être constant pour tous les échantillons testés, il doit être défini de façon à correspondre à l'effort de serrage minimal introduit dans l'assemblage: $F_{0}=F_{0}^{-}$.

Le choix d'un niveau de serrage élevé doit être évité car il ne permet pas de mettre en évidence et/ou comparer efficacement la tenue des produits testés.

Exemple-Effort de serrage suivant NF E 25-030-1: pour une vis M12 de classe 10,9-frottement $\Delta \mu=0,12$ 0,18-précision de serrage $\mathrm{C} 10, F_{0}{ }^{-}=33,6 \mathrm{kN}$.

\subsubsection{Longueur serrée}

La longueur serrée doit être constante pour tous les échantillons testés, elle dépend du type d'essai de dévissage/desserrage choisi et de l'assemblage testé (Tab. 1).

Le choix d'une longueur serrée élevée $\left(L_{\mathrm{p}} / d>3\right)$ doit être évité car il ne permet pas de mettre en évidence et/ou comparer efficacement la tenue des produits testés.

\subsubsection{Critère de défaillance}

Le critère de défaillance est défini par une chute de $50 \%$ de la tension initialement introduite $F_{0}$ à l'issue des $n$ cycles. Dans ces conditions, l'effort de serrage minimal résiduel $F_{0}{ }^{\text {min }}$ est alors inférieur ou égal à $50 \%$ de l'effort de serrage initial $F_{0}$ (soit: $F_{0}{ }^{\min } \leq 50 \% F_{0}$ ). Une rotation maximale peut également être définie (exemple rotation supérieure à 1 tour).
Le choix d'un critère basé sur une perte totale de serrage doit être évité car il ne permet pas de mettre en évidence et/ou comparer efficacement la tenue des produits testés.

\section{2 Étape 1-Recherche du niveau de départ par dichotomie}

En essais préliminaires, un escalier à pas variables par dichotomie est effectué sur la configuration testée, avec:

- un niveau initial de sollicitation $S_{1}\left(S_{\mathrm{i}=1}\right)$ de l'ordre de $50 \%$ de la résistance maximale:

- puis par escalier à pas variables dont les niveaux de sollicitation sont les suivants: $S_{i+1}=S_{i} \pm\left|\frac{S_{i-1}+S_{i}}{2}\right|$ avec $i$ compris entre 1 et 4 , si $i=0$ alors $S_{0}=0$ :

- un nombre d'échantillons de 3 au minimum et de 5 au maximum.

Lorsqu'un changement d'état est détecté, i.e. passage de «non-défaillant serré» $\left(F_{0}{ }^{\min }>50 \% F_{0}\right)$ à «défaillant desserré » $\left(F_{0}{ }^{\mathrm{min}} \leq 50 \% \quad F_{0}\right)$ ou inversement, les essais préliminaires sont terminés (Fig. 12).

Le niveau de départ $S_{0}$ de l'étape 2 (Sect. 3.2) est alors égal à la moyenne des résultats des deux derniers échantillons de l'étape 1.

\section{3 Étape 2-Détermination du niveau de résistance}

Pour déterminer le niveau de résistance $S_{\mathrm{d}}$, les paramètres suivants sont à considérer :

- l'écart entre les niveaux $\left(\Delta S_{\mathrm{d}}\right)$, égal au demi-intervalle entre les deux derniers échantillons de l'étape 1 ;

- le nombre de répétitions : 5 .

Voir exemples en figure 13. Le niveau de résistance $S_{\mathrm{d}}$ est égal à la moyenne des valeurs des 5 échantillons testés en étape 2 et d'un essai fictif. Ce dernier, non physiquement réalisé, a une valeur qui est déterminée par extrapolation du résultat du dernier échantillon testé.

Exemples - Détermination du niveau de l'essai fictif : - dans l'exemple 1b de la figure 13, le dernier échantillon testé de l'étape 2 est «défaillant desserré» donc l'essai fictif est diminué d'un palier ;

- dans l'exemple 2 b de la figure 13, le dernier échantillon testé de l'étape 2 est «non-défaillant serré» donc l'essai fictif est augmenté d'un palier. 


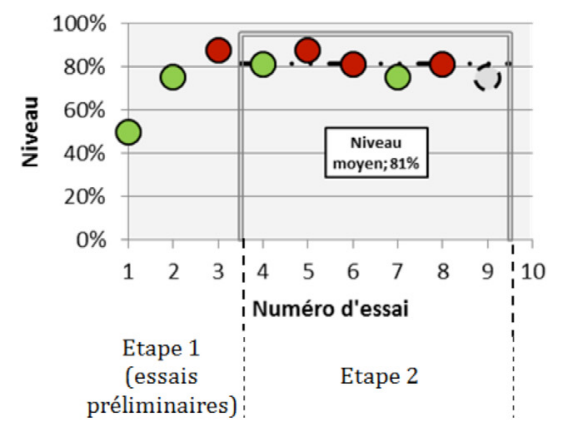

Exemple 1b Légende :
$($ Key) $\stackrel{\text { Ensemble desserré }}{\text { (loose assembly) }}$

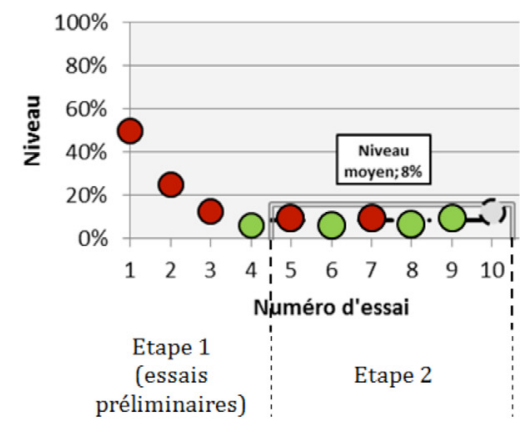

Exemple 2b

Ensemble serré ', Point fictif

(tight assembly) (fictional dot)

Fig. 13. Étape 2-Détermination du niveau de résistance.

Fig. 13. Step 2-Determining the resistance level.

\subsection{Fixations ou dispositifs à essayer}

Afin de réaliser des essais reproductibles, les fixations ou dispositifs doivent être testés dans des conditions identiques. En particulier, vis-à-vis des paramètres décrits dans le paragraphe précédent, ainsi que des pièces de référence éventuellement utilisées, par exemple:

- vis de référence;

- écrou de référence;

- rondelle/plaque de référence, interfaces.

Le type d'essai est à adapter de la manière décrite cidessous, si l'objectif est de:

- déterminer et comparer le niveau de résistance au dévissage/desserrage de différentes fixations ou dispositifs, alors :

- un essai comparatif en conditions représentatives de l'assemblage réel est préconisé ;

- suivre en production la qualité d'un produit vis-à-vis de sa résistance au dévissage/desserrage afin de garantir sa performance, alors :

- un essai de suivi en conditions standardisées est préconisé.

\subsubsection{Essai comparatif en conditions représentatives de l'assemblage réel}

Pour la réalisation d'un essai de ce type, il est nécessaire de tester l'ensemble des éléments de l'assemblage vissé en se rapprochant le plus possible des conditions de service ou d'utilisation.

En particulier :

- l'ensemble des éléments de l'assemblage vissé : vis/écrou/ rondelle ou vis/écrou/matrice taraudée (pas d'utilisation de pièce de référence);

- caractéristiques mécaniques ;

- morphologie;

- traitement de surface;

- rondelle ou plaque d'essai ;

- matériau (acier, alliage d'aluminium...);
- état de surface (rugosité, défaut de forme);

- traitement de surface (revêtement, peinture...);

- lubrification (graisse, huile...);

- collage.

\subsubsection{Essai de suivi en conditions standardisées}

Pour la réalisation d'un essai de ce type, il est nécessaire de tester la fixation avec des pièces de référence identiques.

Les pièces de référence doivent être choisies pour avoir des caractéristiques ou classe de qualité similaires à la fixation testée. Elles doivent être préalablement dégraissées par ultrasons, par exemple conformément au protocole de la norme NF E 25-039.

Afin de sévèriser l'essai ou d'éviter des problèmes de grippage une lubrification peut être réalisée.

\subsection{Résultats d'essai}

Le niveau de résistance au dévissage/desserrage $S_{\mathrm{d}}$ de(s) fixation(s) ou dispositif(s) testé(s) est déterminé par le calcul de la moyenne des résultats des 5 derniers échantillons testés (à l'étape 2).

Si l'objectif et de déterminer le niveau de résistance au dévissage/desserrage de différentes fixations ou dispositifs, alors l'ensemble des résultats permet d'évaluer, en conditions représentatives de l'assemblage réel, les solutions les plus performantes en termes de résistance au dévissage/desserrage $S_{\mathrm{d}}$.

Si l'objectif est de suivre en production la qualité d'un produit, alors les résultats permettent de suivre sa résistance au dévissage/desserrage $S_{\mathrm{d}}$ au cours du temps afin de garantir la constance de sa performance.

\section{Conclusion}

L'ensemble des études menées ces dernières années sur le sujet du dévissage spontané, par le CETIM, ARTEMA Fixations (anciennement AFFIX) et les industriels de la commission R\&D fixations, ont permis de mettre au point deux nouvelles méthodes d'essai : 
- une méthodologie robuste, de type «escalier rapide» ou «modified Staircase» [11], pour la détermination de la résistance au dévissage/desserrage adaptée à tout type d'essai ;

- une méthode d'essai de résistance au dévissage/desserrage par flexion [11], complétant les méthodes déjà existantes avec un autre mode de sollicitation.

Ces deux référentiels sont en cours de normalisation au niveau français et permettront de:

- déterminer et comparer efficacement le niveau de résistance au dévissage/desserrage de différentes fixations ou dispositifs ;

- suivre en production la qualité d'un produit vis-à-vis de sa résistance au dévissage/desserrage afin de garantir sa performance.

Aujourd'hui, aucun essai de suivi de production ou de réception ne permet de vérifier directement la résistance au dévissage/desserrage des produits. Par exemple, les essais de contrôle des couples d'autofreinage (cf. ISO 2320) permettent de contrôler uniquement la fonction d'imperdabilité du produit et non pas sa fonction de résistance au dévissage/desserrage.

\section{References}

1. C. Delcher, Synthèse des études sur l'indesserrabilité, Rapport performance CETIM n 9 Q250, 2014

2. S. Kasei, M. Sawai, On thread-loosening test by means of staircase method, J. Japan Soc. Precision Eng. 49(12), 16631668 (1983)

3. G.H. Junker, New criteria for self-loosening of fasteners under vibration, SAE Trans. 78, 314-335 (1969)
4. J.N. Goodier, R.J. Sweeney, Loosening by vibration of threaded fastenings, Mech. Eng. 67, 798-802 (1945)

5. D.P. Hess, K. Davis, Threaded components under axial harmonic vibration, Acoust. Trans. ASME (1996)

6. S.C. Gambrell, Why bolts loosen, Machine Design 40, 163$167(1968)$

7. S.K. Clark, J.J. Cook, Vibratory loosening of bolts, SAE Paper 660432 (1966)

8. T. Sakai, Investigations of bolt loosening mechanisms, Bull. JSME 21(159), 1385-1394 (1978)

9. M.B. Pearce, A study of vibration-resistant fasteners, SAE Paper $\mathbf{7 3 0 8 2 5}$ (1973)

10. N. Sase, S. Koga, et al., Evaluation of anti-loosening scewfastener innovation and its evaluation, J. Mater. Proc. Technol. 56, 321-332 (1996)

11. ISO 12107: 2012, Metallic materials-Fatigue testingStatistical planning and analysis of data

12. VDI 2230 BLATT 1, «Systematische Berechnung Hochbeanspruchter Schraubenver-bindungen Zylindrische Einschraubenverbindungen, VDI Richtlinien, ICS 21.060.10». VDI-Gesellschaft Entwicklung Konstruktion Vertrieb, Fachberuch Konstruktion, Ausschuss Schraubenverbindungen, 2003, pp. 1-169

13. NF E 25-030-1: 2014, Fixations - Assemblages vissés à filetage métrique ISO - Partie 1 : règles de conception pour les assemblages précontraints - Démarche simplifiée

14. NF E 25-030-2: 2014, Fixations - Assemblages vissés à filetage métrique ISO - Partie 2 : règles de conception pour les assemblages précontraints - Démarche complète

15. Calcul des assemblages 2015 vissés - Assemblages chargés axialement - Techniques de l'ingénieur - BM 5561

16. Calcul des assemblages vissés-Assemblages soumis à une charge excentrée. Partie 1-BM 5562

17. Assemblages par éléments filetés. Modélisation et calculBM 5563

Citation de l'article : Christophe Delcher, Nouvelle approche pour la caractérisation du dévissage et desserrage des assemblages vissés, Matériaux \& Techniques 106, 306 (2018) 